ARTICLE

Received 9 Dec 2012 | Accepted 21 May 2013 | Published 24 Jun $2013 \quad$ DOl: 10.1038/ncomms3037

\title{
Antagonism between binding site affinity and conformational dynamics tunes alternative cis-interactions within Shp2
}

Jie Sun 1,2,3, Shaoying Lu², Mingxing Ouyang², Li-Jung Lin², Yue Zhuo², Bo Liu², Shu Chien4, Benjamin G. Neel ${ }^{5,6}$ \& Yingxiao Wang $1,2,3,7,8$

Protein functions are largely affected by their conformations. This is exemplified in proteins containing modular domains. However, the evolutionary dynamics that define and adapt the conformation of such modular proteins remain elusive. Here we show that cis-interactions between the $\mathrm{C}$-terminal phosphotyrosines and $\mathrm{SH} 2$ domain within the protein tyrosine phosphatase Shp2 can be tuned by an adaptor protein, Grb2. The competitiveness of two phosphotyrosines, namely pY542 and pY580, for cis-interaction with the same SH2 domain is governed by an antagonistic combination of contextual amino acid sequence and position of the phosphotyrosines. Specifically, pY580 with the combination of a favourable position and an adverse sequence has an overall advantage over pY542. Swapping the sequences of pY542 and pY580 results in one dominant form of cis-interaction and subsequently inhibits the trans-regulation by Grb2. Thus, the antagonistic combination of sequence and position may serve as a basic design principle for proteins with tunable conformations.

\footnotetext{
${ }^{1}$ Department of Molecular and Integrative Physiology, University of Illinois at Urbana-Champaign, Urbana, Illinois 61801, USA. ${ }^{2}$ Department of Bioengineering, University of Illinois at Urbana-Champaign, Urbana, Illinois 61801, USA. ${ }^{3}$ Beckman Institute for Advanced Science and Technology, University of Illinois at Urbana-Champaign, Urbana, Illinois 61801, USA. ${ }^{4}$ Department of Bioengineering and Medicine, University of California, San Diego, La Jolla, California 92093, USA. ${ }^{5}$ Campbell Family Cancer Research Institute, Ontario Cancer Institute, Princess Margaret Hospital, University Health Network, Toronto, Ontario, Canada M5G 2M9. ${ }^{6}$ Department of Medical Biophysics, University of Toronto, Toronto, Ontario, Canada M5G 2M9.

${ }^{7}$ Neuroscience Program, Center for Biophysics and Computational Biology, Institute for Genomic Biology, University of Illinois at Urbana-Champaign, Urbana, Illinois 61801, USA. ${ }^{8}$ Department of Bioengineering, University of California, San Diego, La Jolla, California 92093, USA. Correspondence and requests for materials should be addressed to Y.W. (email: yiw015@eng.ucsd.edu).
} 
ntramolecular interactions within proteins (cis-interactions) and allosteric regulations by neighbouring molecules (transinteractions) are common ways to regulate protein functions. One mode of cis-interaction is autoinhibition in which a cisregulatory element interacts with and inhibits the catalytic/ functional domain. Disruption of the cis-interaction usually involves a neighbouring molecule trans-interacting with the cisregulatory element and relieving the autoinhibition to activate the protein $^{1}$. In these interactions, modularity is a recurring theme to regulate diverse functions of proteins ${ }^{2}$. While the specific sequence and position of DNA cis-regulatory elements have been intensively studied to elucidate their roles in regulating gene expression ${ }^{3-5}$, the basic principles underlying how protein modules are designed and arranged to govern their conformations and functions remain largely elusive. X-ray crystallography, NMR spectroscopy and single-molecule spectroscopic methods can provide details of molecular conformations in vitro, but have limited capability in live cells, particularly for proteins containing multiple interacting domains and capable of adopting diverse conformations ${ }^{6}$. Genetically encoded reporters based on fluorescent proteins (FPs) and fluorescence resonance energy transfer (FRET) allow the monitoring of the molecular interactions and conformational changes in live cells with high spatiotemporal resolution ${ }^{7-10}$. In this study, we employed FRET to study the cis- and trans-interactions of Src homology 2 domain-containing protein tyrosine phosphatase 2 (Shp2).

Encoded by PTPN11, Shp2 is a protein tyrosine phosphatase (PTP) that has a critical role in various pathophysiological processes $^{11-13}$. Mutations of PTPN11 cause Noonan Syndrome ${ }^{14}$, LEOPARD syndrome ${ }^{15}$ and juvenile myelomonocytic leukaemia ${ }^{16,17}$. Structurally, Shp2 consists of two Src homology 2 (SH2) domains (N-SH2 and $\mathrm{C}-\mathrm{SH} 2$ ), a PTP domain, and a C-terminal tail (C-tail) containing two major tyrosine sites (Y542 and Y580) (ref. 18). By incorporating non-hydrolysable phosphonomethylene phenylalanine at either Y542 or Y580, it has been suggested that these unnatural chemical modifications mimicking phosphotyrosines may cause the coupling between intramolecular domains in vitro to alter phosphatase activity of Shp2 (ref. 19). However, it is unclear how the protein modules within Shp2 are designed for the cis-regulation and coordination of physiological Shp2 functions in live cells.

We have developed a FRET-based reporter to visualize conformational changes of Shp2 and their underlying regulation mechanisms in live cells. Our results indicate that an antagonistic combination of contextual amino acid (aa) sequence and position of Y542 and Y580 within Shp2 facilitates the effective tuning of Shp2 cis-interactions by Grb2-mediated trans-regulation. The disruption of this antagonistic combination results in the inhibition of trans-regulation of Shp2 conformations and reprograms kinetics of the downstream extracellular signalregulated kinase (ERK) signalling. We suggest that this antagonistic combination of sequence and position may serve as a basic design principle for proteins with tunable conformations and functions.

\section{Results}

FRET-based reporter reveals cis-interaction of Shp2 in vitro. We studied the Shp2 conformations by engineering FRET reporters containing a full-length wild-type (WT) Shp2 flanked by a sensitive FRET pair, an enhanced cyan FP (ECFP) and a yellow FP variant (YFP for energy transfer (YPet) $)^{20}$ (Fig. 1a). The purified WT reporter in vitro can be phosphorylated by platelet-derived growth factor receptor (PDGFR) $\beta$ kinase at both Y542 and Y580 (Fig. 1b), concomitant with an enhanced FRET level represented by an increase in YPet and a decrease in ECFP emission (Fig. 1c). The sustained FRET increase of the WT reporter suggests that PDGFR $\beta$ kinase activity is dominant over intrinsic phosphatase activity of the Shp2 reporter (Fig. 1d). Consistently, the incubation with extra amount of kinase did not cause further increase of the overall level of FRET signals (Supplementary Fig. S1a). In contrast, PDGFR $\beta$ caused the phosphorylation but not FRET change of a mixture containing ECFP-Shp2 (ECFP fused to Shp2) and Shp2-YPet (YPet fused to Shp2) (Supplementary Fig. S1b), indicating minimal intermolecular interactions (trans-interactions) between different copies of Shp2 reporters. Furthermore, the PDGFR $\beta$-induced FRET increase was lost in the YDF reporter with mutations of both Y542 and Y580 (Fig. 1d), which still has intact $\mathrm{SH} 2$ domains capable of trans-interaction with the phosphorylated Y1009 of PDGFR $\beta$ in the assay ${ }^{21}$. Therefore, the FRET response of WT reporter cannot be attributed to its interaction with PDGFR $\beta$ either, and must arise from intramolecular interactions (cis-interactions) within Shp2.

C-SH2 dominates the cis-interaction in vitro. The PDGFR $\beta$ induced FRET response was eliminated by YDF or the double SH2 domain mutant (RDL) (Fig. 1d), suggesting that the cis-interactions within Shp2 are mediated by its $\mathrm{SH} 2$ domains and phosphotyrosines at C-tail. Single C-SH2 mutation (R138L), but not N-SH2 mutation (R32L), eliminated the PDGFR $\beta$-induced FRET increase (Fig. 1e), indicating that $\mathrm{C}-\mathrm{SH} 2$ is essential for the cis-interaction. Y580F, but not Y542F mutation, blocked most FRET increase (Fig. 1f), even though the phosphorylation of these two mutants was similar to that of WT reporters (Fig. 1g). Therefore, the FRET response of WT reporter in vitro is mainly due to the cis-interaction between C-SH2 and phosphorylated Y580 (pY580) (Fig. 1h). This notion is further supported by the obvious FRET response of a mutant disrupting the functions of both N-SH2 and Y542 (R32LY542F) (Supplementary Fig. S1c).

cis-Interaction competitiveness of the phosphotyrosines. Interestingly, there was a consistent although minor FRET response of the Y580F reporter (Fig. If and inset), suggesting that phosphorylated Y542 (pY542) may also undergo cis-interaction with C-SH2 in the absence of pY580 but only yield a weak FRET change. The reason for this weak FRET may come from the 51aalong flexible C-tail region of the Y580F reporter between pY542 (the C-SH2-binding site) and YPet (the FRET acceptor) (Fig. 2a), in contrast to the short 13aa-region between pY580 and YPet in WT and Y542F reporters (Fig. 1h). Indeed, truncating this 51aalong region $(\Delta 580)$ to 13 aa (Fig. 2 a) caused a substantial FRET increase upon PDGFR $\beta$ incubation (Fig. 2b), which can be abolished by additional C-SH2 (R138L) but not N-SH2 (R32L) mutation (Supplementary Fig. S1d). These results confirmed that the cis-interaction between pY542 and C-SH2 can occur in the absence of pY580 (Fig. 2a). However, pY580 dominates the cisinteractions with $\mathrm{C}-\mathrm{SH} 2$ in the population of WT reporters because the conversion of weak-FRET population, if any, to high FRET by Y542F mutation did not cause any significant increase in the overall FRET response (Fig. 1d,f,h). As both tyrosine sites are phosphorylated in the WT reporter (Fig. 1g), we suspect that the aa sequence surrounding pY580 has a higher affinity binding to C-SH2. Surprisingly, direct affinity measurement of the isolated pY542 or pY580 peptide towards C-SH2 by surface plasmon resonance (SPR) revealed a much higher binding affinity between pY542 and C-SH2 (Fig. 2c and Table 1). Furthermore, when the Y542 sequence was truncated so that it was replaced by the relocated Y580 sequence $(\Delta 542$, Fig. $2 a)$, no FRET response was observed (Fig. 2b), although substantial phosphorylation of Y580 
a

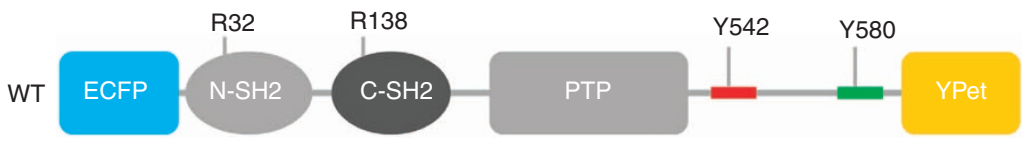

b

$$
\operatorname{PDGFR\beta }(\min ) \quad 0 \quad 15 \quad 30 \quad 45 \quad 60 \quad 120 \quad 180
$$

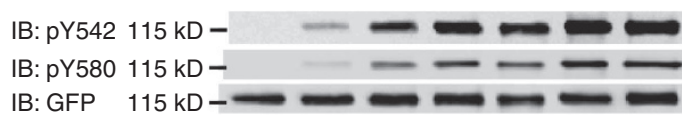

d

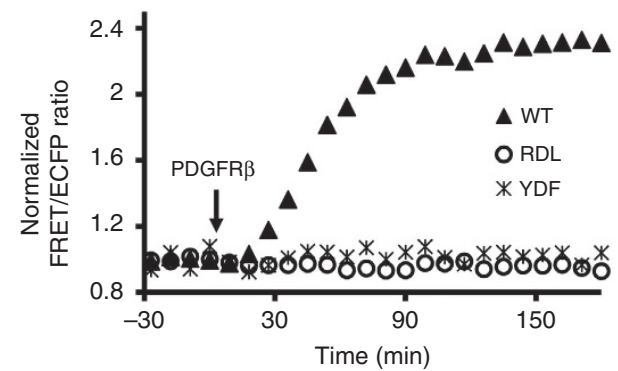

c
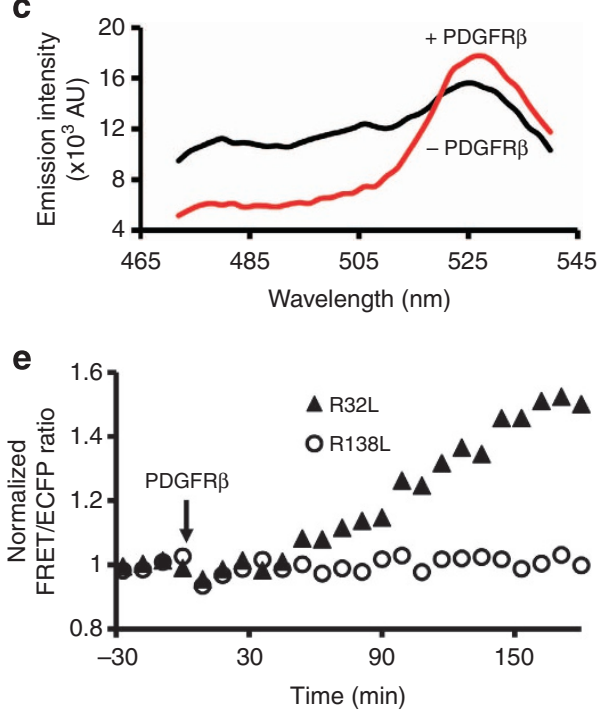

f

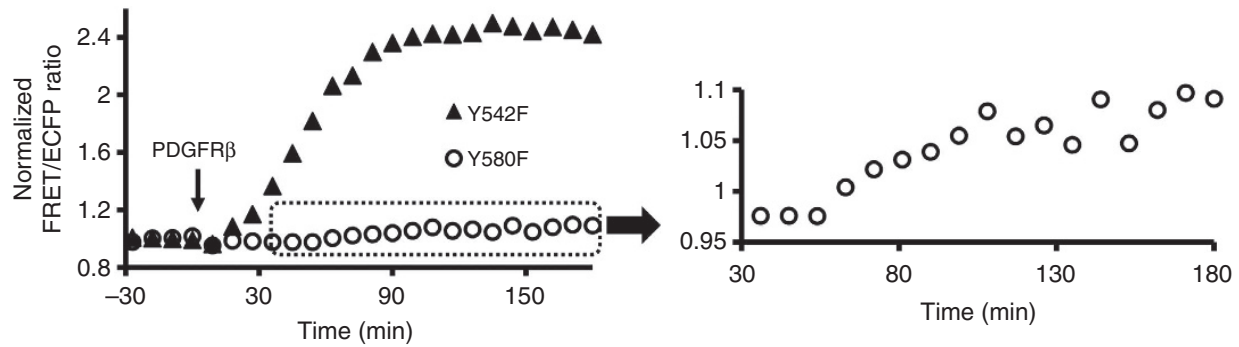

g
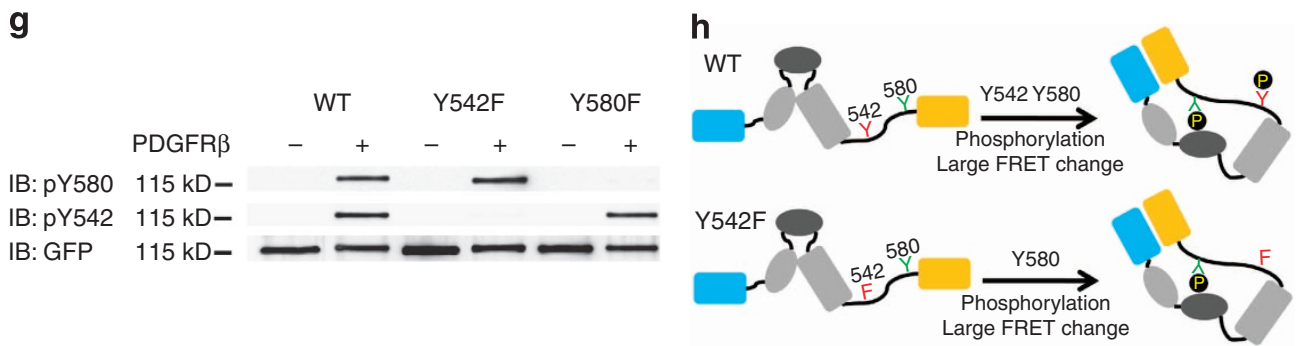

Figure 1 | cis-Interactions of the Shp2 reporters in vitro. (a) Schematic drawing of the WT Shp2 reporter, with mutation sites as indicated. Y542 and Y580 sequences are coloured in red and green, respectively. (b) The phosphorylation of the WT reporter upon PDGFR $\beta$ incubation in vitro (refer to Supplementary Fig. S6a for the whole blots). (c) The emission spectrum change of the WT reporter before (black) and after (red) PDGFR $\beta$ incubation. (d-f) The ratio time courses of the WT reporter and its mutants as indicated. (f) Inset shows the time course of zoom-in ratios of Y580F reporter upon PDGFR $\beta$ incubation. (g) The tyrosine phosphorylation of Y580 and Y542 of the WT reporter, its Y542F or Y580F mutant in vitro before and after PDGFR $\beta$ incubation. (h) The models depict the cis-interactions and FRET changes of the WT and Y542F reporters upon phosphorylation in vitro.

$A U$, arbitrary unit.

occurred (Supplementary Fig. S1e). As the only difference between $\Delta 580$ and $\Delta 542$ reporters is their distinct sequences at position 542 (Fig. 2a,b), the stronger FRET response of $\Delta 580$ reporter with pY542 sequence confirms that C-SH2 also favours the pY542 sequence over pY580 in the context of the whole Shp2 protein. The dominant cis-interaction between pY580 and C-SH2 within the WT reporter where both Y542 and Y580 are phosphorylated, therefore, suggests that position 580 may be favoured over position 542 for $\mathrm{C}-\mathrm{SH} 2$ interaction to overpower the advantageous pY542 sequence. Indeed, when the reporter was reengineered to have identical Y542 sequence at both 542 and 580 positions (Y542Y542), position 580 is the preferred cis-interaction site for C-SH2 because a mutation at position 580 but not 542 suppressed the majority of FRET response (Fig. 2d). Therefore, the dominant cis-interaction between $\mathrm{C}-\mathrm{SH} 2$ and pY580 within the WT reporter in vitro is resulted from an antagonistic combination of an adverse sequence but a favourable position at pY580.

Different conformational regulation of Shp2 in cells. Both purified WT reporter and full-length Shp2 in vitro showed an 
a
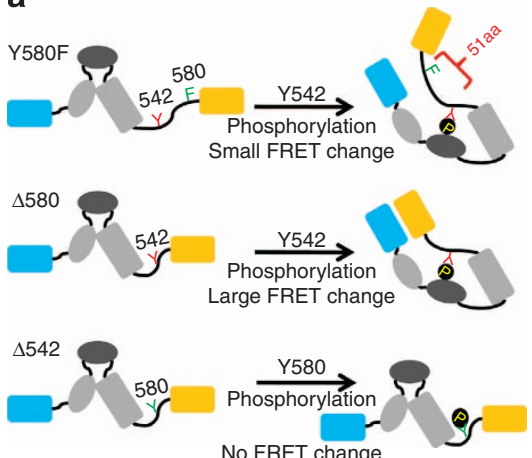

b
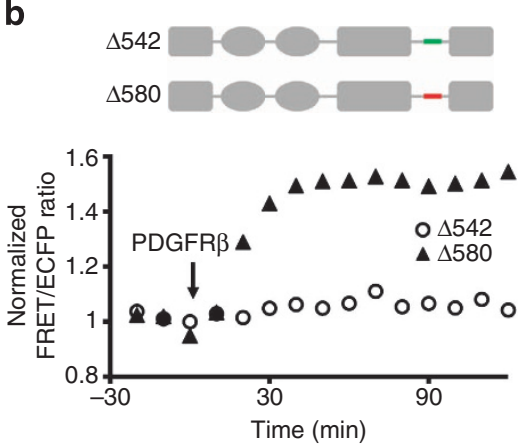

C

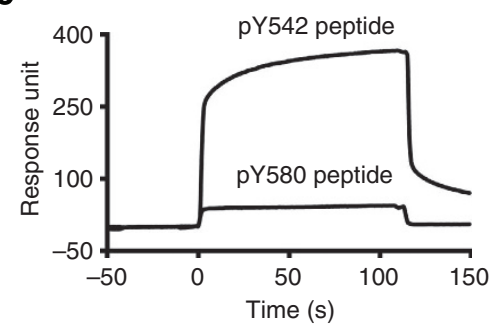

d
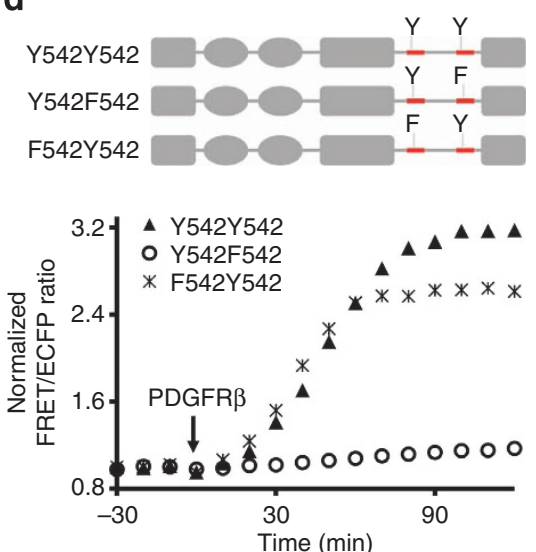

Figure 2 | The combination of contextual sequence and position determines the preference of phosphotyrosines for cis-interaction. (a) The models depict the cis-interactions of the $\mathrm{Y} 580 \mathrm{~F}, \Delta 580$ and $\Delta 542$ reporters upon phosphorylation in vitro. (b) The ratio time courses of $\Delta 542$ and $\Delta 580$ reporters. Y542 and Y580 sequences are coloured in red and green, respectively. (c) The binding curves of the C-SH2 domain (4.5 $\mu$ M) towards immobilized pY542 or pY580 peptides measured by SPR (refer to Supplementary Fig. S8 for the binding kinetics and original sensorgrams). (d) The ratio time courses of engineered Y542Y542 and its mutant reporters as indicated.

Table 1 | The dissociation constant $\left(K_{d}\right)$ of different peptides towards the binding of Grb2 or C-SH2 domain of Shp2.

\begin{tabular}{llcc} 
Peptide name & Peptide sequence & C-SH2 $\boldsymbol{K}_{\mathbf{d}}(\boldsymbol{\mu} \mathbf{M})$ & Grb2 $\boldsymbol{K}_{\mathbf{d}}(\mathbf{n M} \mathbf{)}$ \\
\hline pY542 & KGHEPYTNIKYS & $15.1 \pm 0.47$ & $118 \pm 10.3$ \\
pY580 & SARVpYENVGLM & $294 \pm 23.6$ & $68.5 \pm 8.7$ \\
\hline${ }^{\star}$ All peptides contain a biotin-ASASA at their N termini. & \\
\hline
\end{tabular}

increased phosphatase activity upon incubation with PDGFR $\beta$, which can interact with the $\mathrm{N}-\mathrm{SH} 2$ domain and, therefore, unmask the phosphatase domain to activate Shp2 (Supplementary Fig. S2a). When expressed in mouse embryonic fibroblasts (MEFs), the WT reporter was recruited to membrane ruffles after platelet-derived growth factor (PDGF) stimulation with similar localization and C-tail phosphorylation as the endogenous Shp2 (Supplementary Fig. S2b,c). Therefore, the Shp2 reporter has the same functional activation mechanism and localizations as the endogenous Shp2. We hence applied the Shp2 reporter to monitor Shp2 conformation regulations in mammalian cells. We confirmed that the cellular concentration of Shp2 reporter when expressed in mammalian cells to be in the range of micromolar, which is similar to the concentration used in vitro (Supplementary Fig. S2d). PDGF caused a FRET increase of the WT reporter in MEFs (Fig. 3a, Supplementary Fig. S3a, and Supplementary Movie 1), concomitant with phosphorylation at both Y542 and Y580 (Supplementary Fig. S2c). In contrast, no FRET response can be observed upon PDGF stimulation in MEFs expressing both ECFP-Shp2 and Shp2-YPet (Supplementary Fig. S3b), confirming the cis-interaction within WT reporter for the FRET change in live cells. Consistent with our in vitro results, RDL, YDF or R138L, but not R32L mutation, abolished the FRET response (Fig. 3b, Supplementary Fig. S3c). Surprisingly, in marked contrast to their effects in vitro, Y542F but not Y580F mutation, eliminated the PDGF-induced FRET response in MEFs (Fig. 3b, Supplementary Fig. S3c, Supplementary Movies 2 and 3). The cis-interaction between pY542 and C-SH2 is hence dominant in MEFs, in contrast to the pY580 and C-SH2 pair in vitro. Consistently, the Y580F reporter, with its Y542 intact and phosphorylated (Fig. 3c), had a similar level of FRET change as that of WT reporter in MEFs (Fig. 3b). The $\Delta 580$ reporter also had stronger FRET responses than the WT reporter in MEFs, which was abolished by additional R138L but not R32L mutation (Supplementary Fig. S3d). All these results confirmed the primary cis-interaction between pY542 and C-SH2 in MEFs.

Y580 phosphorylation depends on Y542 and C-SH2 in MEFs. We further explored the molecular mechanism underlying the distinct conformational regulations of Shp2 in vitro and in MEFs. Y580 phosphorylation in the absence of Y542 was significantly inhibited in MEFs (Fig. 3c) (ref. 22), in contrast to the independence of Y580 and Y542 phosphorylations in vitro (Fig. 1g). The phosphorylation of Y542 is hence essential for and may occur before Y580 phosphorylation within each copy of the reporters in MEFs. Indeed, Y542 sequence fused to green fluorescent protein (GFP) was phosphorylated substantially faster than Y580 sequence in vitro (Fig. 3d). R138L mutant to disrupt C-SH2 also inhibited the phosphorylation of Y580, but not that of Y542 in MEFs (Fig. 3e). Hence, pY542 and its subsequent cisinteraction with C-SH2 can precede and mediate the phosphorylation of Y580 in MEFs. 
a

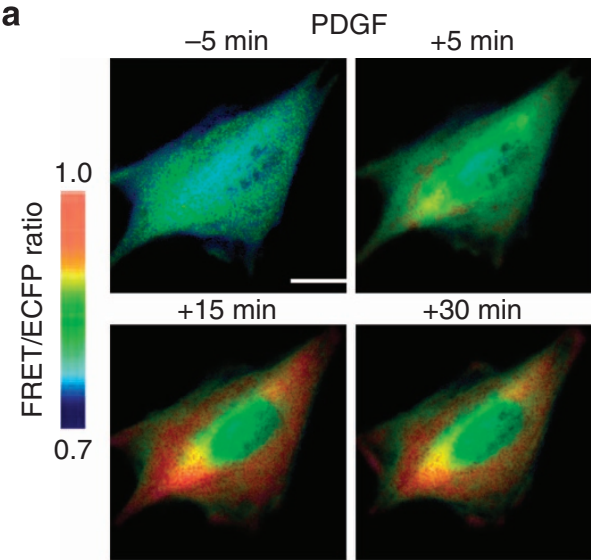

b
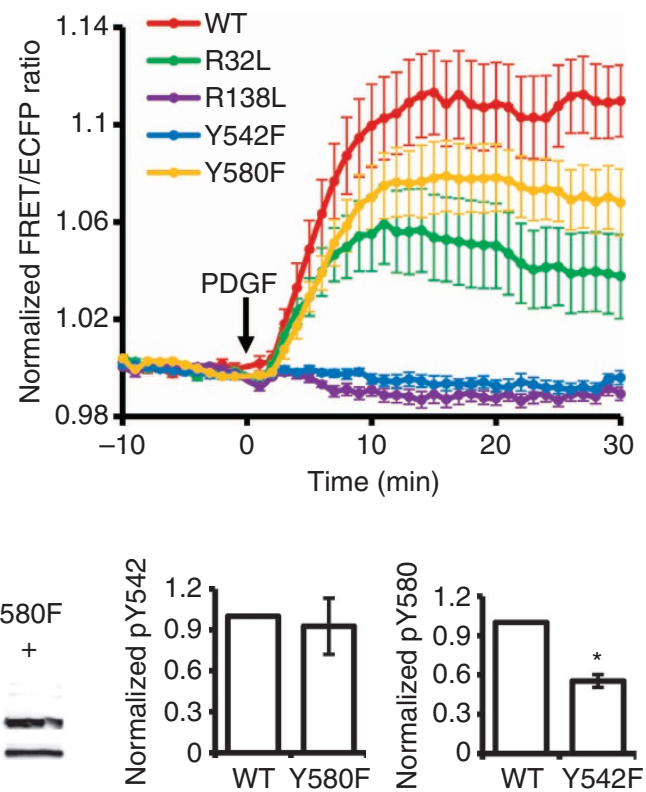

d
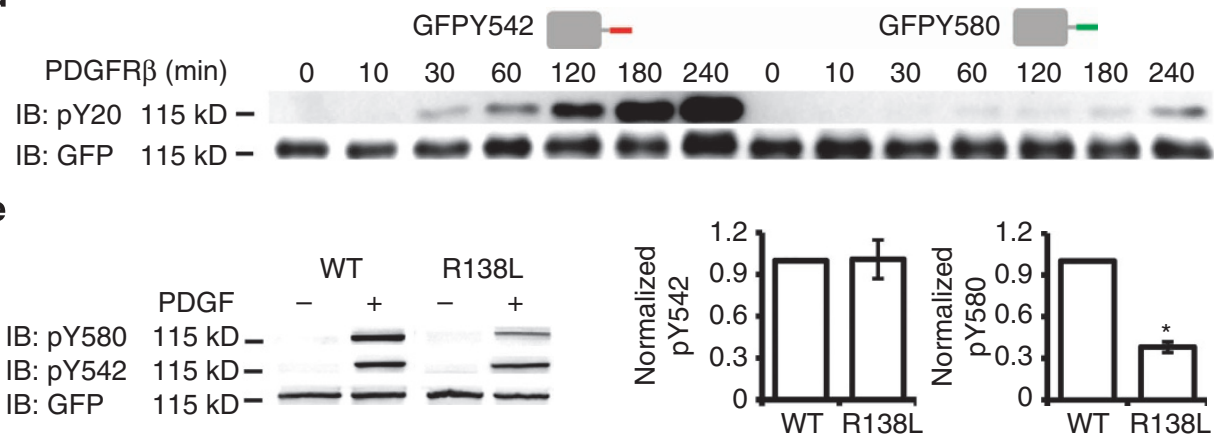

Figure 3 | cis-Interactions and their regulation within the Shp2 reporters in MEFs. (a) The ratiometric images of a MEF cell transfected with the WT reporter before and after PDGF stimulation for various periods of time. Scale bar, $10 \mathrm{~mm}$. (b) The ratio time courses (mean \pm s.e.m., $n=18,17,15,15$ and 11$)$ of MEFs expressing the WT reporter or its mutants. (c) The phosphorylation of WT and tyrosine mutant reporters in MEFs before and after 5 min PDGF stimulation. Bar graphs represent the mean \pm s.e.m. ${ }^{*}, P<0.05$. (Student's $t$-test, $n=3$, refer to Supplementary Fig. S6b for the whole blots). (d) The tyrosine phosphorylation levels of the purified GFPY542 $(1 \mu \mathrm{M})$ or GFPY580 $(1 \mu \mathrm{M})$ reporter before and after $10 \mathrm{nM}$ PDGFR $\beta$ incubation for indicated time periods. (e) The phosphorylation of WT and C-SH2 mutant reporters in MEFs before and after 5 min PDGF stimulation. Bar graphs represent the mean \pm s.e.m. ${ }^{\star}, P<0.05$ (Student's $t$-test, $n=3$ ). Whole-cell lysate was used for panels $\mathbf{c}, \mathbf{e}$.

Grb2 tunes cis-interactions and FRET levels of Shp2 reporter. We then examined whether the phosphorylation of Y580, in the absence of pY542, can be rescued in MEFs to allow its cis-interaction with C-SH2 as it does in vitro. When PDGFR $\beta$ was overexpressed in MEFs, Y580 phosphorylation can occur in the absence of pY542 (Fig. 4a) to cause C-SH2/pY580 interaction and result in a considerable FRET change upon PDGF stimulation (Fig. 4b). It was puzzling then why the C-SH2/pY580 cis-interaction dominates in vitro but not in MEFs when both Y542 and Y580 are phosphorylated in the WT reporter. As the phosphorylation of Y580 follows and is dependent on the cis-interaction of $\mathrm{C}-\mathrm{SH} 2 / \mathrm{pY} 542$ in MEFs, we hypothesized that molecules interacting with Shp2 in MEFs, such as Grb2 (ref. 23), may sequester the more potent pY580 from competing for the pY542-occupied $\mathrm{C}-\mathrm{SH} 2$. Indeed, purified Grb2 can reduce and tune the FRET levels when added to the phosphorylated $\mathrm{Y} 542 \mathrm{~F}$ reporter in vitro (Fig. 4c), by interacting with the available pY580 in the reporter (Fig. 4d). The amount of Grb2 can apparently cause a proportional reduction in overall FRET signals by decreasing the percentage of reporters adopting high-FRET cis-interactions (Fig. 4c,d). This tuning ability of Grb2 is mediated by its $\mathrm{SH} 2$ domain as the SH2-disabling mutant Grb2RV was no longer effective (Fig. 4e). Consistently, overexpression of Grb2 with varying concentrations can proportionally tune, via its $\mathrm{SH} 2$ domain, the FRET level of the Y542F reporter in MEFs overexpressing constitutively active PDGFR (Tel-PDGFR $\beta)^{24}$ (Fig. 5a,b). Therefore, Grb2 can trans-interact with and sequester pY580 from cis-interaction with $\mathrm{C}-\mathrm{SH} 2$ in $\mathrm{Y} 542 \mathrm{~F}$ reporter. Further results showed that Grb2 can also tune the FRET signals of WT reporter in MEFs (Fig. 5c). However, it is unclear whether Grb2 preferentially trans-interacts with pY580 when both Y542 and Y580 are intact and phosphorylated in the WT reporter. SPR results revealed that Grb2 has a significantly higher affinity binding to the isolated pY580 peptide than to the pY542 peptide (Table 1). In addition, R138L mutation disrupting the C-SH2 occupation of pY542 in the Y580F reporter significantly increased the pY542-Grb2 interaction (Fig. 5d), suggesting that the preformed cis-interaction of $\mathrm{C}-\mathrm{SH} 2$ and 
a

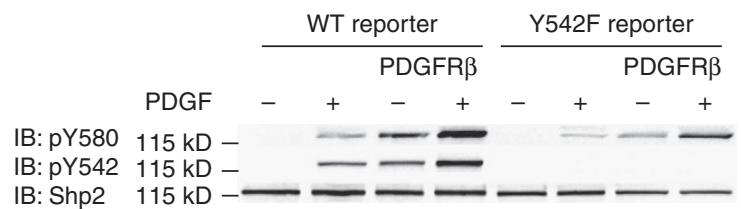

b

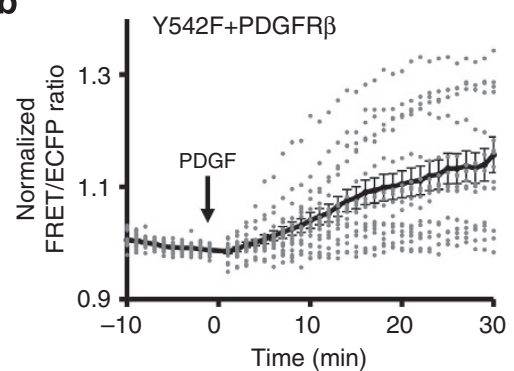

d

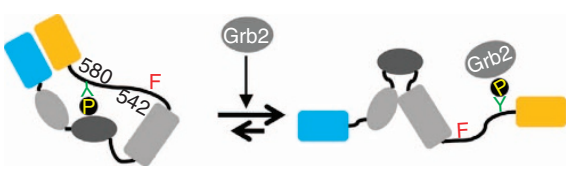

C

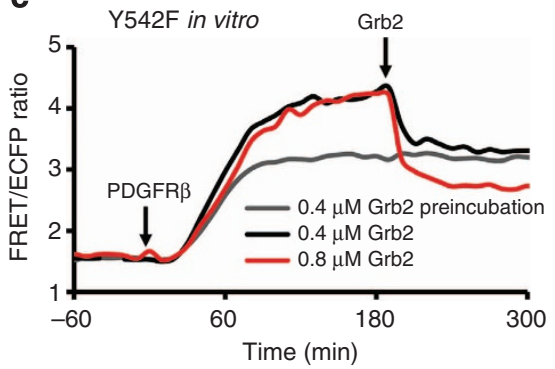

e

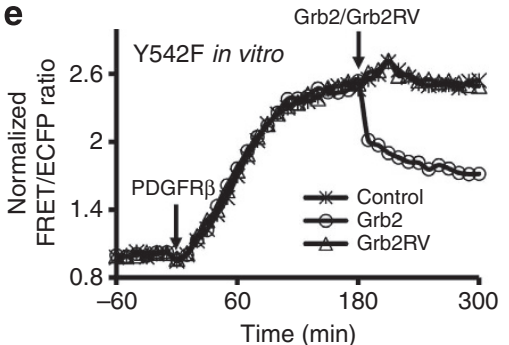

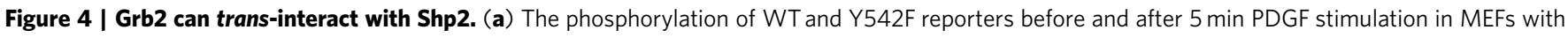
or without the overexpression of PDGFR 3 . (b) The ratio time courses of Y542F reporter before and after PDGF stimulation in MEFs overexpressing PDGFR $\beta$. The dotted lines from each cell are overlaid with the solid curve (mean \pm s.e.m.). (c) The PDGFR $\beta$-induced ratio changes of Y542F reporter pre- or post-incubated with Grb2 in vitro. (d) The model depicts the effect of Grb2 in altering the Y542F reporter conformations. (e) The ratio time courses of the Y542F reporter in vitro upon PDGFR $\beta$ incubation, followed by the addition of Grb2 or Grb2RV.

pY542 can reduce the availability of pY542 for Grb2 and hence promote Grb2-pY580 interaction in the WT reporter. Indeed, pY542 in the Y580F reporter allowed much less Grb2 binding comparing with the WT reporter (Fig. 5e), confirming that pY580 in Shp2 is the preferred and primary trans-interaction site for Grb2. This Grb2-pY580 association can sequester the free pY580 from competing for the pY542-occupied C-SH2, which should in turn stabilize the pY542/C-SH2 complex in MEFs (Fig. 5f).

Rewired Shp2 reprograms cis-interaction and ERK dynamics. Based on the results, it is clear that the Y542 contextual sequence is favoured as a substrate for PDGFR and has a higher affinity for $\mathrm{C}-\mathrm{SH} 2$ upon phosphorylation. However, position 580 is favoured by $\mathrm{C}-\mathrm{SH} 2$ for the cis-interaction over position 542 within the context of the intact Shp2 molecule. This antagonistic combination of sequence and position results in a favoured cis-interaction between C-SH2 and Y580 in vitro, where both Y542 and Y580 are independently phosphorylated by the PDGFR in solution. In MEFs, the recruitment of Shp2 to the membrane receptor PDGFR through its $\mathrm{N}-\mathrm{SH} 2$ domain ${ }^{25}$ leads to an early phosphorylation of the favoured substrate Y542, which subsequently undergoes cis-interaction with C-SH2. This pY542/C$\mathrm{SH} 2$ interaction and the resulted conformational change are essential and facilitate the phosphorylation of Y580, which recruits Grb2 to sequester the otherwise favoured pY580 from competing for the pY542-occupied C-SH2. As such, Grb2 in MEFs can bind to pY580 and shift the equilibrium of cis-interaction towards pY542/C-SH2, distinct from the dominant pY580/C-SH2 cis-interaction of Shp2 in solutions.
It hence becomes apparent that the moderate difference between pY542 and pY580 in competing for C-SH2, resulting from the antagonistic combination of tyrosine sequence and position, can allow easier alteration of Shp2 cis-interactions by Grb2 binding. We have then rewired the modular arrangement within Shp2 to develop a SWAP reporter by swapping the Y542 and Y580 sequences to combine the favourable Y542 sequence with the preferred 580 position in a synergistic fashion (Y542': Y580 sequence at 542 position; Y580': Y542 sequence at 580 position), widening the gap of competitiveness between the two tyrosines (Fig. 6a).

We thereafter examined the characteristics of the SWAP reporter in vitro and in MEFs. Y542' and Y580' of the SWAP reporter were phosphorylated independently both in vitro and in MEFs (Fig. 6b). PDGF also induced a remarkably high FRET change of the SWAP reporter in MEFs, significantly stronger than that of the WT reporter and with a level as high as the in vitro response (Supplementary Fig. S4a,b; Fig. 3b, Supplementary Movie 4). More importantly, Y580'F or R138L, but not Y542'F nor R32L, abolished the FRET response of the SWAP reporter both in vitro and in MEFs (Fig. 6c,d; Supplementary Fig. S4c,d; Supplementary Movie 5 and 6). These results confirmed that the SWAP reporter loses the tunable cis-interactions with its C-SH2 undergoing one form of cis-interaction with the pY580' both in vitro and in MEFs. The Y542' phosphorylation of SWAP reporter was also lower than that of WT reporter (Supplementary Fig. S4e), suggesting that the regulatory mechanism of promoting Y580 phosphorylation by C-SH2/pY542 cis-interaction no longer exists in the SWAP reporter. Consequently, this rewired cis-regulation of Shp2 conformation caused 

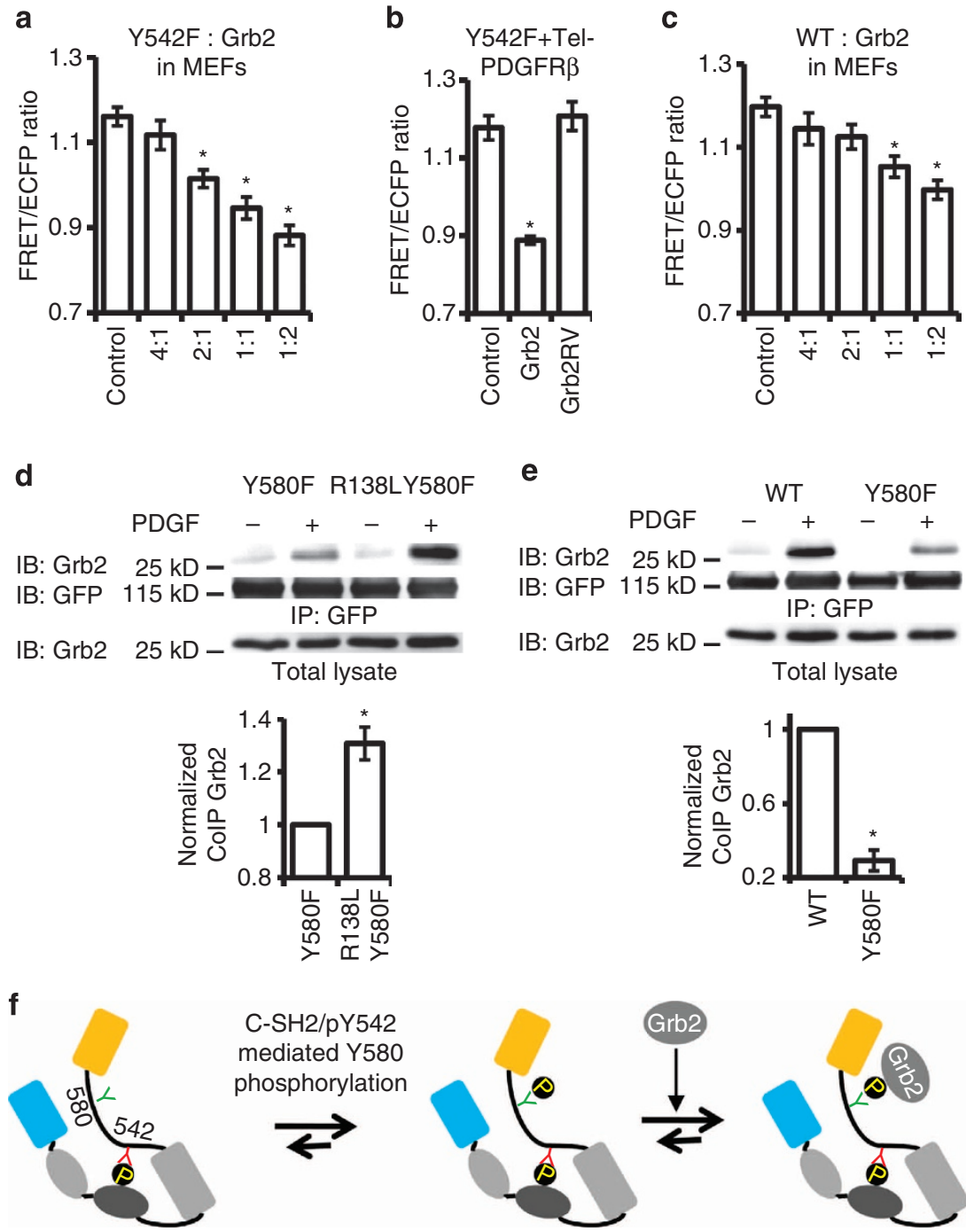

Figure 5 | Grb2 tunes the cis-interaction of Shp2 in MEFs. (a-c) The averaged ratios of the Y542F (a,b) or WT (c) reporter in MEFs coexpressing Tel-PDGFR $\beta$ with control vector, different amounts of Grb2 (a,c), or Grb2RV (b). (a) $n=16,17,14,18,11,{ }^{\star}, P<4.55 \mathrm{e}-5$; (b) $n=38,25,26,{ }^{\star}, P<1.0 \mathrm{e}-8$; (c) $n=10,20,18,15,35,{ }^{*} P<3.1 e-4$ (Student's t-test with Bonferroni correction). (d-e) The amount of Grb2 coimmunoprecipitated with WT or its mutant reporters in MEFs before and after 15 min PDGF stimulation. ${ }^{*}, P<0.05$ (Student's $t$-test, $n=3$ ). Bar graphs represent the mean \pm s.e.m. of normalized intensity against its corresponding control group (refer to Supplementary Fig. S7a for the whole blots). (f) The model depicts the early interaction of C-SH2/pY542, the subsequent pY580 phosphorylation which recruits Grb2 and is sequestered by Grb2 to stabilize this C-SH2/pY542 pair.

a significantly more transient ERK phosphorylation upon PDGF stimulation (Fig. 6e, Supplementary Fig. S4f). Therefore, by swapping the Y542 and Y580 sequences, we have rewired the regulation mechanism of Shp2 conformation, which led to a reprogrammed downstream ERK signalling.

\section{Discussion}

Our results indicate that a simple antagonistic combination of contextual sequence and position can result in tunable cisinteractions within Shp2, leading to complex regulation routes and functional outcomes. pY542 in the WT reporter has the combination of favoured sequence and adverse position while pY580 has adverse sequence and favoured position. Swapping the sequences results in a synergistic combination: pY580' with favoured sequence and position while pY542' with adverse sequence and position (Fig. 6a). As such, the order of these four phosphotyrosines in terms of their affinity for C-SH2 is as follows: pY542'<pY542<pY580<pY580'. Therefore, the antagonistic combination has two layers of effect on the cis-interactions of Shp2. First, it ensures that the affinity of both pY542 and pY580 in WT Shp2 for cis-regulation is moderate enough to allow effective trans-regulations and prevent possibly insurmountable cis-interactions as in the case of pY580'. This note is supported by the observation that Grb2 more effectively inhibit the FRET response of Y542 reporter (pY580 and C-SH2 cis-interaction) than that of Y542'FSWAP (pY580' and C-SH2 cis-interaction) (Supplementary Fig. S5a). In addition, the affinity of Grb2 towards isolated pY542 or pY580 peptide is several orders higher than that of C-SH2 (Table 1). This higher affinity of Grb2 allows the effective competitiveness of trans-interaction, overcoming the entropic advantage of cis-interactions within the WT Shp2 (ref. 26). Second, the antagonistic combination can lead to a relatively small difference in the cis-interaction competitiveness between the two phosphotyrosines than the synergistic combination (pY580-pY542<pY580'-pY542'). Therefore, the presence of Grb2 in mammalian cells can tune the cis-interaction of WT Shp2 towards pY542/C-SH2 pair from 
a

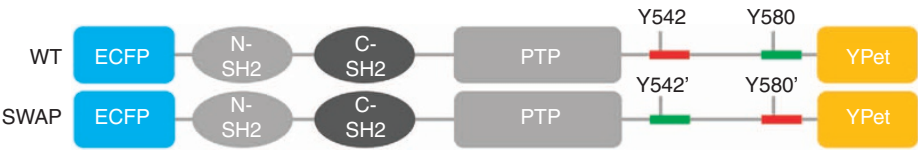

b
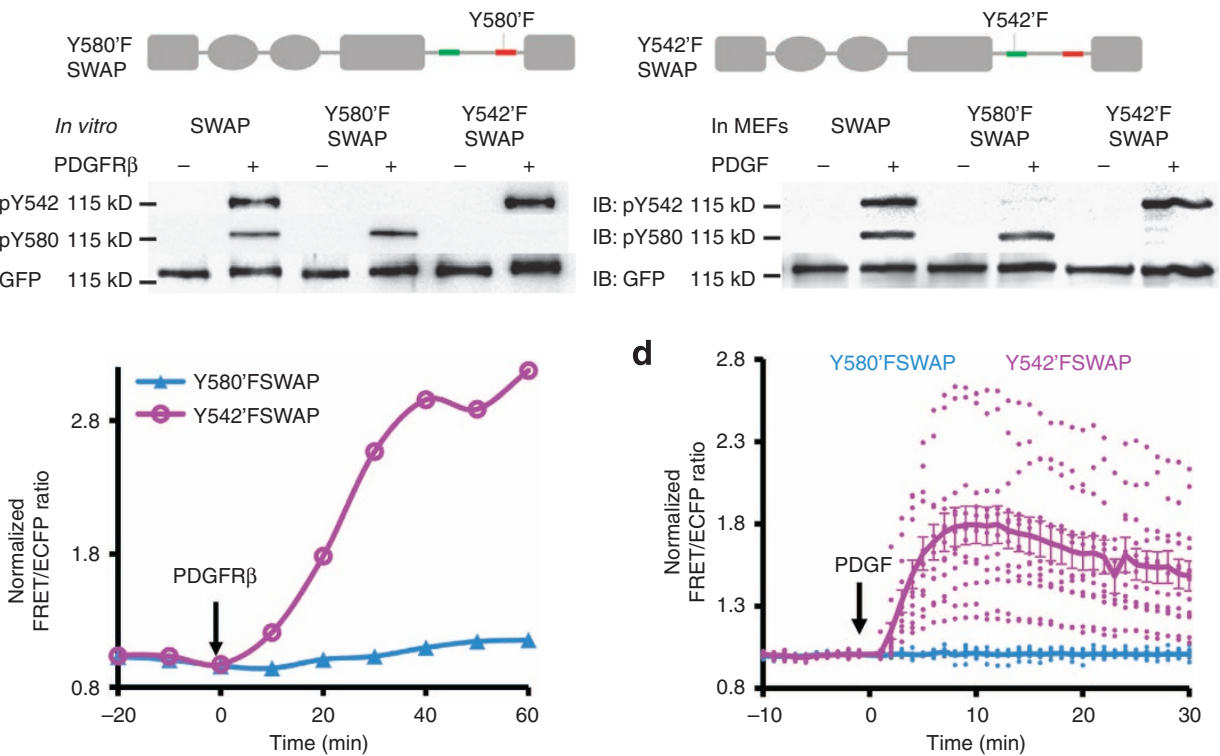

e
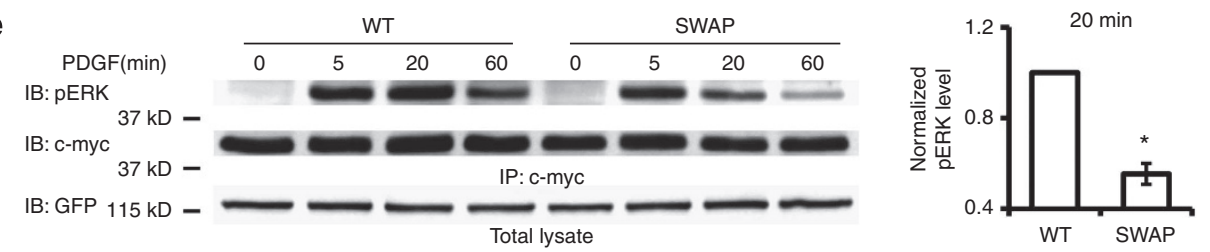

Figure 6 | The loss of tunable cis-interaction within the SWAP reporter and its effect on ERK phosphorylation. (a) Schematic drawings and comparison of the WT and SWAP Shp2 reporters. Y542 and Y580 sequences are coloured in red and green, respectively. (b) The phosphorylation of SWAP and its mutant reporters in vitro (left) or in MEFs (right, whole-cell lysate). (c,d) The ratio changes of Y542'FSWAP and Y580'FSWAP reporters (c) in vitro or (d) in MEFs. (e) The ERK phosphorylation at different time points (left) and its quantification (mean \pm s.e.m.) at 20 min (right) upon PDGF stimulation in MEFs expressing either WT or SWAP reporter as indicated. ${ }^{\star}, P<0.05$ (Student's $t$-test, $n=3$, refer to Supplementary Fig. S7b for the whole blots).

pY580/C-SH2 pair. In contrast, the pY580'/C-SH2 pair in SWAP reporter dominates both in cells and in vitro. As such, the synergistic combination in the SWAP reporter resulted in the enlargement of the difference in competitiveness between the two phosphotyrosines, the inhibition of Grb2-mediated trans-regulation, the loss of tunable cis-interactions within Shp2 and eventually the reprogramming of downstream ERK signalling dynamics. This combinatorial effect of protein modules is strikingly similar to that of DNA cis-regulatory elements on regulating a coordinated gene expression where sequence-specific promoters/enhancers are highly structured and precisely positioned ${ }^{4,5,27}$. We suggest that this antagonistic combination of protein sequence and position can serve as a basic design principle for the construction of tunable protein elements and building blocks to develop higher order and programmable molecular machineries, capable of learning and adapting according to environmental cues.

Systems and synthetic biologists have long employed networks to describe the signalling transduction, with each node representing one molecule and its determined function ${ }^{28}$. We discovered in this work that a tunable regulation mechanism is embedded within one molecule, for the processing of environmental inputs to result in differential Shp2 conformations and functional outcomes. These cis-interactions within Shp2 allow the fine regulation of protein functions beyond the simple disruption of autoinhibition for protein activations. This protein-based regulation can also produce fast responses at specific subcellular locations, surpassing the capacity of gene-based tuning of cellular functions ${ }^{29}$. Hence, this tunable regulation mechanism built in Shp2 may provide a prominent example on how nature can design/evolve molecules with their conformations/ functions differentially regulated under different subcellular environments. Integrating with molecular switches/gates based on scaffold proteins ${ }^{30,31}$ and genetic circuits ${ }^{29}$, the understanding and rewiring of these modular motif arrangements may also provide powerful means to reprogram the downstream signalling cascades at subcellular levels ${ }^{28}$. It is of note that the tunable cis-interactions within Shp2 may not exist in lower organisms as the Drosophila ortholog, Corkscrew, only retains Y542 while the Caenorhabditis elegans ortholog, Ptp-2, lacks both tyrosines ${ }^{18}$. Therefore, this cis-regulatory mechanism of Shp2 may represent a distinguished evolutionary innovation in higher organisms for the fine-tuning of functions resulting in higher levels of organism complexity ${ }^{32}$. In addition, our results show that Grb2 can tune the cis-interaction of Shp2. However, Grb2 may not be the only molecule that can trans-regulate Shp2 function, as Grb2 short interfering RNA (siRNA) knockdown did not increase the FRET level of Y542F reporter compared with control siRNA (Supplementary Fig. S5b). 
Our FRET-based Shp2 reporter mainly revealed the cis-interactions between phosphotyrosines and C-SH2, but not $\mathrm{N}-\mathrm{SH} 2$. It appears unlikely that $\mathrm{N}-\mathrm{SH} 2$ has cis-interactions with pY542 or pY580 as the binding cleft of N-SH2 domain in the fulllength Shp2 is in a compressed and closed conformation limiting accessibility for phosphopeptides ${ }^{33,34}$. It has been established that the phosphotyrosine interaction of $\mathrm{N}-\mathrm{SH} 2$ can drastically affect the Shp 2 phosphatase activity by relieving the autoinhibitory cis-interaction between $\mathrm{N}-\mathrm{SH} 2$ and PTP domain ${ }^{35,36}$. In our study, the binding of C-SH2 by either pY542 or pY580 did not have significant impact on the phosphatase activity as the phosphatase activity of R32L reporter did not increase after being phosphorylated by PDGFR kinase (Supplementary Fig. S5c,d). Therefore, the SH2 domain interactions within Shp2 can have two main functional outcomes: the trans-interaction of $\mathrm{N}-\mathrm{SH} 2$ regulates enzymatic phosphatase activity, whereas that cis-interaction of C-SH2 controls the Y580 phosphorylation and the subsequent Grb2 recruitment to govern downstream signalling cascades, such as ERK phosphorylation.

While the initial phosphorylation of ERK is mainly dependent on the receptor-Ras pathway ${ }^{37}$, the phosphorylation of Y580 is crucial for the maintenance of a sustained ERK phosphorylation $^{22}$. Indeed, it has been proposed that pY580 may serve as a docking site for Grb2/Sos complex and, therefore, regulate downstream ERK pathway ${ }^{38}$. In the SWAP reporter, pY542' was also constitutively lower (Supplementary Fig. S4e), causing a transient ERK phosphorylation (Fig. 6e). As the different dynamics of ERK activation has been shown to determine cell fate and long-term memory ${ }^{39,40}$, the wiring and rewiring of Y542 and Y580 positions in Shp2 to control pY580/ pY542' and hence ERK phosphorylation dynamics can have profound impact on pathophysiological consequences.

The modular SH2-phosphotyrosine trans-interaction between different proteins has been well established to mediate molecular interactions and signalling transduction ${ }^{41}$. The intramolecular cis-interaction of $\mathrm{SH} 2$-phosphotyrosine has also been shown to regulate the molecular functions ${ }^{42}$. For example, the cis-interaction between pY527 and SH2 domain of Src kinase can cause the autoinhibition of Src kinase domain ${ }^{43}$. The SH2phosphotyrosine trans-interaction can further compete for the cis-interaction of domains within a molecule and modulate the molecular function. Indeed, Src SH2 domain can interact with pY397 at FAK to release the FERM domain from masking the FAK kinase domain and cause FAK activation ${ }^{44}$. However, most studies involve only one pair of SH2-phosphotyrosine interaction. The Shp2 molecule has two coordinating pairs of $\mathrm{SH} 2$-phosphotyrosine interaction. Our results suggest that an antagonistic combination of sequence and position of the phosphorylated tyrosines can result in tunable cis-interactions within one molecule to allow effective trans-regulations of its conformational changes and subsequently functions. With the prevalence of $\mathrm{SH} 2$-phosphotyrosine and other modules, the complexity of cis-interactions between multiple interacting domains revealed by our FRET-based study may be a typical but overlooked layer that contributes to the overall complexity of cell signalling.

\footnotetext{
Methods

Reporter construction and other constructs. The Shp2 reporter was constructed by fusing a full-length human Shp2 complementary DNA to an N-terminal ECFP sequence and a C-terminal YPet sequence in pRSETb (Invitrogen) for bacterial expression. For mammalian cell expression, the Shp2 reporter was cloned into pcDNA3.1 (Invitrogen) behind a Kozak sequence using BamHI/EcoRI restriction sites. Point mutations of the Shp2 reporters were generated using QuikChange SiteDirected Mutagenesis Kit (Agilent Technologies). Deletion or insertion mutations of Shp2 reporter were generated by PCR and subsequent ligation. The sequence of
} $11 \mathrm{aa}(\mathrm{s})$ surrounding Y542 or Y580 (including four residues in front of and six residues behind tyrosine) was referred as Y542 or Y580 sequence, respectively. The $\Delta 542$ reporter has a truncation between residue 538 and 575 , whereas the $\Delta 580$ reporter has a truncation between residue 556 and 593. The SWAP reporter has the 11 aa sequences surrounding Y542 and Y580 exchanged. The Y542Y542 reporter has the Y542 sequence at both Y542 and Y580 positions. Tel-PDGFR $\beta$ constructs were from Dr Gary Gilliland.

Protein expression and purification. HEK cells (ATCC) transfected with WT or mutated Shp2 reporters were washed with cold PBS and then lysed in buffer containing $50 \mathrm{mM}$ Tris- $\mathrm{HCl} \mathrm{pH} 7.5,100 \mathrm{mM} \mathrm{NaCl}, 1 \mathrm{mM}$ EDTA, $0.2 \mathrm{mM}$ phenylmethylsulphonyl fluoride, $0.2 \%$ Triton $\mathrm{X}-100$ and a protease inhibitor cocktail tablet (Roche). Lysates were centrifuged at $10,000 \mathrm{~g}$ at $4{ }^{\circ} \mathrm{C}$ for $10 \mathrm{~min}$. Supernatants were incubated with Ni-NTA agarose beads (Qiagen), which were then washed with $50 \mathrm{mM}$ Tris- $\mathrm{HCl} \mathrm{pH} 7.5,100 \mathrm{mM} \mathrm{NaCl}, 10 \mathrm{mM}$ imidazole.

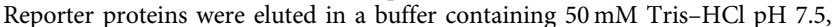
$100 \mathrm{mM} \mathrm{NaCl}$ and $100 \mathrm{mM}$ imidazole. Alternatively, reporter proteins were purified from $E$. coli for large quantities. Reporters in pRSETb were transformed into BL21 and the expression was induced by exposure to $0.4 \mathrm{mM}$ isopropyl- $\beta$-Dthiogalactoside for $16 \mathrm{~h}$ at room temperature (RT). The bacteria were lysed in buffer A (20 mM imidazole, $20 \mathrm{mM}$ Tris- $\mathrm{HCl} \mathrm{pH} \mathrm{8,} 200 \mathrm{mM} \mathrm{NaCl})$ with EmulsiFelx-B15 (Avestin). Lysates were centrifuged at $10,000 \mathrm{~g}$ at $4{ }^{\circ} \mathrm{C}$ for $20 \mathrm{~min}$. Supernatants were then loaded onto a HisTrap HP column using an AKTA purifier FPLC system (GE). The column was subsequently washed with $20 \mathrm{ml}$ of buffer A and the fusion protein eluted using a $20-400 \mathrm{mM}$ imidazole concentration gradient developed in $50 \mathrm{ml}$ buffer $A$ at $0.5 \mathrm{ml} \mathrm{min}^{-1}$. Fractions with absorption at $430 \mathrm{~nm}$ were pooled, concentrated and subsequently loaded onto a HiLoad 16/60 Superdex 200 gel filtration column (GE). Buffer B (30 mM Tris- $\mathrm{HCl}$ pH 8.0, $200 \mathrm{mM} \mathrm{NaCl}$, $1 \mathrm{mM}$ dithiothreitol) was then used for elution. Fractions around molecular weight $120 \mathrm{kDa}$ were collected and concentrated for in vitro assays.

Immunoprecipitation and immunoblotting. Cells were washed three times with ice-cold PBS and lysed in a buffer (Cell Signaling) containing $50 \mathrm{mM}$ Tris- $\mathrm{HCl}$, pH 7.5, $125 \mathrm{mM} \mathrm{NaCl}, 1 \%$ Triton X-100, 0.1\% SDS, $5 \mathrm{mM} \mathrm{NaF}, 1 \mathrm{mM} \mathrm{Na}_{3} \mathrm{VO}_{4}$, $1 \mathrm{mM}$ phenylmethylsulfonyl fluoride and $10 \mu \mathrm{g} \mathrm{ml}^{-1}$ leupeptin. Lysates were centrifuged at $10,000 \mathrm{~g}$ at $4{ }^{\circ} \mathrm{C}$ for $10 \mathrm{~min}$, and the protein concentration of clarified lysates was determined by Bradford assay (Biorad). Antibodies $(1 \mu \mathrm{g}$ per $100 \mu \mathrm{g}$ total lysate) for Co-immunoprecipitation (IP) or IP were incubated with cell lysates for $1 \mathrm{~h}$ at $4{ }^{\circ} \mathrm{C}$. Protein A/G beads (Santa Cruz) were then added to the mixture and incubated overnight at $4{ }^{\circ} \mathrm{C}$. The beads were washed three times with lysis buffer and boiled for $5 \mathrm{~min}$ in SDS protein loading buffer. After centrifugation, the supernatants were resolved by SDS-polyacrylamide gel electrophoresis. The proteins were then transferred onto a nitrocellulose membrane and blocked with $5 \%$ bovine serum albumin in TTBS buffer ( $50 \mathrm{mM}$ Tris- $\mathrm{HCl}, 145 \mathrm{mM} \mathrm{NaCl}, 0.05 \%$ Tween-20, $\mathrm{pH}$ 7.4) for $2 \mathrm{~h}$ at RT. Membranes were further incubated with primary antibodies overnight at $4{ }^{\circ} \mathrm{C}$, washed and then incubated with horseradish peroxidase-conjugated secondary antibodies for $2 \mathrm{~h}$ at RT. Signals were detected using SuperSignal Western Pico or Femto ECL Kit (Pierce). Western results were quantified with ImageJ (NIH). Our statistics were performed with two-tailed Student's $t$-test. When multiple $t$-tests were performed, the $\alpha$-value was modified by the Bonferroni correction, such that $\alpha=0.0125$ for up to four $t$-tests performed in each group (Fig. 5). Anti-phospho-SHP-2 (Tyr-542) and (Tyr-580) antibodies were from Cell Signaling Technology (Cat \# 3751 and 3703). Anti-GFP antibody for IP was from Abcam (Cat \# ab290). Anti-phosphotyrosine pY20 antibody was from BD Transduction laboratory (Cat \# 610000). Anti-Shp2, anti-GFP antibodies for immunoblotting, as well as HRP-conjugated secondary antibodies were purchased from Santa Cruz Biotechnology (Cat \# sc-280, sc-8334, sc-2004, sc-2005).

Image acquisition and analysis. Glass-bottom dishes (Cell E\&G Inc.) were coated with $10 \mu \mathrm{g} \mathrm{ml}^{-1}$ fibronectin (Sigma) overnight at $37^{\circ} \mathrm{C}$. Transfected MEFs were plated onto these dishes overnight in DMEM containing $0.5 \%$ FBS before imaging During imaging, MEFs (ATCC) were maintained in $\mathrm{CO}_{2}$-independent medium (Invitrogen) with $0.5 \% \mathrm{FBS}$ at $37^{\circ} \mathrm{C}$ for $10 \mathrm{~min}$ before treatment with $20 \mathrm{ng} \mathrm{ml}^{-1}$ PDGF (Sigma). Images were obtained on a Zeiss Axiovert inverted microscope equipped with a cooled charge-coupled device camera (Cascade 512B; Photometrics) using MetaFluor 6.2 software (Molecular Devices). The following filter sets (Chroma) were used in our experiments for FRET imaging: a dichroic mirror $(450 \mathrm{~nm})$, an excitation filter $420 / 20 \mathrm{~nm}$, an ECFP emission filter $475 / 40 \mathrm{~nm}$, and a FRET emission filter $535 / 25 \mathrm{~nm}$. The excitation filter for ECFP at $420 \pm 20 \mathrm{~nm}$ was specifically selected to shift towards lower wavelength away from the peak excitation spectrum of ECFP to reduce the cross-excitation of the FRET acceptor YPet, which has significantly higher brightness than ECFP. This filter selection can minimize the effect of bleed-through on the FRET channel. The fluorescence intensity of non-transfected cells was quantified as the background signal and subtracted from the ECFP and FRET signals of transfected cells. The pixel-by-pixel ratio images of FRET/ECFP were calculated based on the background-subtracted fluorescence intensity images of ECFP and FRET by using the MetaFluor software. These ratio images were displayed in the intensity modified display mode in which the colour and brightness of each pixel is determined by the 
FRET/ECFP ratio and ECFP intensity, respectively. The emission ratio were quantified and analysed by Excel (Microsoft).

In vitro kinase assays. Purified proteins were dialyzed overnight at $4{ }^{\circ} \mathrm{C}$ in kinase buffer $\left(50 \mathrm{mM}\right.$ Tris- $\mathrm{HCl} \mathrm{pH} \mathrm{8.0,100} \mathrm{mM} \mathrm{NaCl,} 10 \mathrm{mM} \mathrm{MgCl}_{2}$ and $2 \mathrm{mM}$ dithiothreitol). Fluorescence emission spectra of the purified reporters $(0.5 \mu \mathrm{M})$ were measured in 96-well plates with an excitation wavelength of $430 \mathrm{~nm}$ using a fluorescence plate reader (TECAN, Sapphire II). Emission ratios of acceptor/donor $(526 \mathrm{~nm} / 478 \mathrm{~nm})$ were measured at $30^{\circ} \mathrm{C}$ before and after the addition of $1 \mathrm{mM}$ ATP and 10-100 nM active PDGFR $\beta$ kinases (Millipore).

Affinity measurement by SPR. The binding affinity of pY542 and pY580 peptides to $\mathrm{C}-\mathrm{SH} 2$ domain or Grb2 was assessed by SPR analysis on a BIAcore 3000 instrument (GE). Biotinylated peptides were immobilized on streptavidin-coated SA sensor chips (GE). Before immobilization, the sensor chip was conditioned with a solution containing $1 \mathrm{M} \mathrm{NaCl}$ and $50 \mathrm{mM} \mathrm{NaOH}$. The binding assays were conducted at RT in HBS-EP buffer (GE, $10 \mathrm{mM}$ HEPES, pH 7.4, $150 \mathrm{mM} \mathrm{NaCl}$, $3 \mathrm{mM}$ EDTA, $0.005 \%$ Surfactant P20). Each pY peptide was injected at a flow rate of $5 \mu \mathrm{l} \mathrm{min}{ }^{-1}$ until a constant level of response units (500-600 RU) was obtained. Varying concentrations of histidine-tagged C-SH2 or Grb2 were passed over the immobilized peptides for $2 \mathrm{~min}$. To remove the proteins from the peptide, a regeneration buffer $(10 \mathrm{mM} \mathrm{NaOH}, 200 \mathrm{mM} \mathrm{NaCl}, 0.05 \%$ SDS $)$ was applied. The equilibrium response unit $\left(\mathrm{RU}_{\mathrm{eq}}\right)$ at a given protein concentration was obtained by subtracting the response of a blank control chip. The dissociation constants $\left(K_{\mathrm{d}}\right)$ were calculated by the BIAevaluation software using the equation 1, where $\mathrm{RU}_{\mathrm{eq}}$ is the measured response unit at a given $\mathrm{SH} 2$ domain concentration, and $\mathrm{RU}_{\max }$ is the maximum response unit:

$$
\mathrm{RU}_{\mathrm{eq}}=\mathrm{RU}_{\max }[\mathrm{SH} 2] /\left(K_{\mathrm{d}}+[\mathrm{SH} 2]\right)
$$

Immunostaining. Cells were washed three times with ice-cold PBS and fixed with $4 \%$ paraformaldehyde at RT for $15 \mathrm{~min}$ before they were permeabilized with $0.1 \%$ TritonX-100 in PBS for $30 \mathrm{~min}$. After that, the cells were rinsed and blocked with $1 \%$ BSA in PBS for $20 \mathrm{~min}$. They were then incubated with primary antibody (Santa Cruz, Cat \# sc-280 or sc-8994, 1:1,000 dilution) in PBS at RT for $2 \mathrm{~h}$ before they were incubated with secondary antibody conjugated with fluorescence dye at RT for $1 \mathrm{~h}$. After another round of washing, the cells were subjected to imaging under microscopy.

Phosphatase activity assays. Phosphatase activity was measured using fluorogenic 6,8-difluoro-4-methylumbelliferyl phosphate (DiFMUP; Invitrogen) as the substrate. Each reaction contained $50 \mathrm{mM}$ Tris pH 8, $100 \mathrm{mM} \mathrm{NaCl}, 10 \mathrm{mM} \mathrm{MgCl}_{2}$, $2 \mathrm{mM}$ dithiothreitol, $20 \mu \mathrm{M}$ DiFMUP and $20 \mathrm{nM}$ Shp2 reporter in a total reaction volume of $100 \mu \mathrm{l}$ in a well of 96 -well plates. Reactions were initiated by the addition of DiFMUP, followed by incubation for $30 \mathrm{~min}$ at $30^{\circ} \mathrm{C}$. The fluorescence signal of the reaction product, 6,8-difluoro-4-methylumbelliferone, was measured at an excitation wavelength of $355 \mathrm{~nm}$ and an emission of $460 \mathrm{~nm}$ with a plate reader.

\section{References}

1. Pufall, M. A. \& Graves, B. J. Autoinhibitory domains: modular effectors of cellular regulation. Annu. Rev. Cell. Dev. Biol. 18, 421-462 (2002).

2. Bhattacharyya, R. P., Remenyi, A., Yeh, B. J. \& Lim, W. A. Domains, motifs, and scaffolds: the role of modular interactions in the evolution and wiring of cell signaling circuits. Annu. Rev. Biochem. 75, 655-680 (2006).

3. Woody, S. T., Fong, R. S. \& Gussin, G. N. Effects of a single base-pair deletion in the bacteriophage lambda PRM promoter. Repression of PRM by repressor bound at OR2 and by RNA polymerase bound at PR. J. Mol. Biol. 229, 37-51 (1993).

4. Cox, 3rd R. S., Surette, M. G. \& Elowitz, M. B. Programming gene expression with combinatorial promoters. Mol. Syst. Biol. 3, 145 (2007).

5. Murphy, K. F., Balazsi, G. \& Collins, J. J. Combinatorial promoter design for engineering noisy gene expression. Proc. Natl Acad. Sci. USA 104, 12726-12731 (2007).

6. Sakon, J. J. \& Weninger, K. R. Detecting the conformation of individual proteins in live cells. Nat. Methods 7, 203-205 (2010).

7. Kiyokawa, E., Hara, S., Nakamura, T. \& Matsuda, M. Fluorescence (Forster) resonance energy transfer imaging of oncogene activity in living cells. Cancer Sci. 97, 8-15 (2006).

8. Wang, Y., Shyy, J. Y. \& Chien, S. Fluorescence proteins, live-cell imaging, and mechanobiology: seeing is believing. Annu. Rev. Biomed. Eng. 10, 1-38 (2008).

9. Aye-Han, N. N., Ni, Q. \& Zhang, J. Fluorescent biosensors for real-time tracking of post-translational modification dynamics. Curr. Opin. Chem. Biol. 13, 392-397 (2009).

10. Lam, A. J. et al. Improving FRET dynamic range with bright green and red fluorescent proteins. Nat. Methods 9, 1005-1012 (2012).

11. Tonks, N. K. Protein tyrosine phosphatases: from genes, to function, to disease. Nat. Rev. Mol. Cell. Biol. 7, 833-846 (2006).
12. Bard-Chapeau, E. A. et al. Ptpn11/Shp2 acts as a tumor suppressor in hepatocellular carcinogenesis. Cancer Cell 19, 629-639 (2011).

13. Chan, G., Kalaitzidis, D. \& Neel, B. G. The tyrosine phosphatase Shp2 (PTPN11) in cancer. Cancer Metastasis. Rev. 27, 179-192 (2008).

14. Tartaglia, M. et al. Mutations in PTPN11, encoding the protein tyrosine phosphatase SHP-2, cause Noonan syndrome. Nat. Genet. 29, 465-468 (2001).

15. Tartaglia, M. \& Gelb, B. D. Noonan syndrome and related disorders: genetics and pathogenesis. Annu. Rev. Genomics Hum. Genet. 6, 45-68 (2005).

16. Loh, M. L. et al. Mutations in PTPN11 implicate the SHP-2 phosphatase in leukemogenesis. Blood 103, 2325-2331 (2004).

17. Tartaglia, M. et al. Somatic mutations in PTPN11 in juvenile myelomonocytic leukemia, myelodysplastic syndromes and acute myeloid leukemia. Nat. Genet. 34, 148-150 (2003).

18. Neel, B. G., Gu, H. \& Pao, L. The 'Shp'ing news: SH2 domain-containing tyrosine phosphatases in cell signaling. Trends Biochem. Sci. 28, 284-293 (2003).

19. Lu, W., Gong, D., Bar-Sagi, D. \& Cole, P. A. Site-specific incorporation of a phosphotyrosine mimetic reveals a role for tyrosine phosphorylation of SHP-2 in cell signaling. Mol. Cell. 8, 759-769 (2001).

20. Ouyang, M., Sun, J., Chien, S. \& Wang, Y. Determination of hierarchical relationship of Src and Rac at subcellular locations with FRET biosensors. Proc. Natl Acad. Sci. USA 105, 14353-14358 (2008).

21. Eck, M. J., Pluskey, S., Trub, T., Harrison, S. C. \& Shoelson, S. E. Spatial constraints on the recognition of phosphoproteins by the tandem $\mathrm{SH} 2$ domains of the phosphatase SH-PTP2. Nature 379, 277-280 (1996).

22. Araki, T., Nawa, H. \& Neel, B. G. Tyrosyl phosphorylation of Shp2 is required for normal ERK activation in response to some, but not all, growth factors. J. Biol. Chem. 278, 41677-41684 (2003).

23. Li, W. et al. A new function for a phosphotyrosine phosphatase: linking GRB2 Sos to a receptor tyrosine kinase. Mol. Cell. Biol. 14, 509-517 (1994).

24. Carroll, M., Tomasson, M. H., Barker, G. F., Golub, T. R. \& Gilliland, D. G. The $\mathrm{TEL} /$ platelet-derived growth factor beta receptor (PDGF beta R) fusion in chronic myelomonocytic leukemia is a transforming protein that self-associates and activates PDGF beta R kinase-dependent signaling pathways. Proc. Natl Acad. Sci. USA 93, 14845-14850 (1996).

25. Nakata, S. et al. Regulation of platelet-derived growth factor receptor activation by afadin through SHP-2: implications for cellular morphology. J. Biol. Chem. 282, 37815-37825 (2007).

26. Nguyen, J. T. \& Lim, W. A. How Src exercises self-restraint. Nat. Struct. Biol. 4, 256-260 (1997)

27. Levine, M. \& Tjian, R. Transcription regulation and animal diversity. Nature 424, 147-151 (2003).

28. Lim, W. A. Designing customized cell signalling circuits. Nat. Rev. Mol. Cell. Biol. 11, 393-403 (2010).

29. Lu, T. K., Khalil, A. S. \& Collins, J. J. Next-generation synthetic gene networks Nat. Biotechnol. 27, 1139-1150 (2009).

30. Bashor, C. J., Helman, N. C., Yan, S. \& Lim, W. A. Using engineered scaffold interactions to reshape MAP kinase pathway signaling dynamics. Science 319, 1539-1543 (2008).

31. Good, M. C., Zalatan, J. G. \& Lim, W. A. Scaffold proteins: hubs for controlling the flow of cellular information. Science 332, 680-686 (2011).

32. Lim, W. A. \& Pawson, T. Phosphotyrosine signaling: evolving a new cellular communication system. Cell 142, 661-667 (2011).

33. Darian, E., Guvench, O., Yu, B., Qu, C. K. \& MacKerell, Jr A. D. Structural mechanism associated with domain opening in gain-of-function mutations in SHP2 phosphatase. Proteins 79, 1573-1588 (2011).

34. Guvench, O., Qu, C. K. \& MacKerell, Jr A. D. Tyr66 acts as a conformational switch in the closed-to-open transition of the SHP-2 N-SH2-domain phosphotyrosine-peptide binding cleft. BMC Struct. Biol. 7, 14 (2007).

35. Sugimoto, S., Wandless, T. J., Shoelson, S. E., Neel, B. G. \& Walsh, C. T. Activation of the SH2-containing protein tyrosine phosphatase, SH-PTP2, by phosphotyrosine-containing peptides derived from insulin receptor substrate-1. J. Biol. Chem. 269, 13614-13622 (1994).

36. Hof, P., Pluskey, S., Dhe-Paganon, S., Eck, M. J. \& Shoelson, S. E. Crystal structure of the tyrosine phosphatase SHP-2. Cell 92, 441-450 (1998).

37. Egan, S. E. et al. Association of Sos Ras exchange protein with Grb2 is implicated in tyrosine kinase signal transduction and transformation. Nature 363, 45-51 (1993).

38. Dance, M., Montagner, A., Salles, J. P., Yart, A. \& Raynal, P. The molecular functions of Shp2 in the Ras/Mitogen-activated protein kinase (ERK1/2) pathway. Cell Signal. 20, 453-459 (2008).

39. Santos, S. D., Verveer, P. J. \& Bastiaens, P. I. Growth factor-induced MAPK network topology shapes Erk response determining PC-12 cell fate. Nat. Cell. Biol. 9, 324-330 (2007).

40. Pagani, M. R., Oishi, K., Gelb, B. D. \& Zhong, Y. The phosphatase SHP2 regulates the spacing effect for long-term memory induction. Cell 139, 186-198 (2009). 
41. Pawson, T., Gish, G. D. \& Nash, P. SH2 domains, interaction modules and cellular wiring. Trends Cell Biol. 11, 504-511 (2001).

42. Boggon, T. J. \& Eck, M. J. Structure and regulation of Src family kinases. Oncogene 23, 7918-7927 (2004).

43. Brown, M. T. \& Cooper, J. A. Regulation, substrates and functions of src. Biochim. Biophys. Acta. 1287, 121-149 (1996).

44. Mitra, S. K., Hanson, D. A. \& Schlaepfer, D. D. Focal adhesion kinase: in command and control of cell motility. Nat. Rev. Mol. Cell. Biol. 6, 56-68 (2005).

\section{Acknowledgements}

We thank Professor Gary Gilliland (Harvard University) for the Tel-PDGFR $\beta$ construct. This work is supported by grants from NIH HL098472, CA139272, NSF CBET0846429 (Y.W.) and NIH R37 CA49132, the Ontario Ministry of Health and Long Term Care, and the Princess Margaret Hospital Foundation (B.G.N.).

\section{Author contributions}

J.S., S.L., S.C. and Y.W. designed the experiments. J.S., M.O., L.L., Y.Z., and B.L performed the experiments. J.S. and S.L. analysed the data. J.S., B.G.N, S.C. and Y.W. wrote the manuscript.

\section{Additional information}

Supplementary Information accompanies this paper at http://www.nature.com/ naturecommunications

Competing financial interests: The authors declare no competing financial interests.

Reprints and permission information is available online at http://npg.nature.com/ reprintsandpermissions/

How to cite this article: Sun, J. et al. Antagonism between binding site affinity and conformational dynamics tunes alternative cis-interactions within Shp2. Nat. Commun. 4:2037 doi: 10.1038/ncomms3037 (2013). 